\title{
Screening Programs for Cervical Cancer: Investigation "SWOT" in Calabria
}

\author{
Giuseppe Andrea De Biase ${ }^{1}$, Caterina Azzarito ${ }^{1}$, Carmela Bianchi ${ }^{2}$, Amalia De Luca ${ }^{2}$, Domenico Gullà ${ }^{1}$, Salvatore \\ Lopresti $^{1}$, Dario Macchione ${ }^{1}$, Domenica Mignuolo ${ }^{1}$, Liliana Rizzo ${ }^{1}$ and Filomena Zappia ${ }^{3}$ \\ 1. Health Protection and Health Policy Department, Calabria Region \\ 2. Company Provincial Health of Cosenza 87100 \\ 3. Company Provincial Health of Reggio Calabria 89100
}

\begin{abstract}
Screening programs for cervical cancer: a "SWOT" investigation in the Calabria Region)—The low adherence to screening programs for cervical cancer in Calabria, was analyzed by performing a SWOT analysis with concerned health professionals. The survey shows that screening organized in Calabria is active and well regulated, the human and financial resources are scarce and the healthcare management has paid little attention to the prevention of women's cancers. In the perspective of a necessary improvement of the regional system of screening, it would be appropriate to enable promotion strategies and more effective communication, overcoming possible cultural factors hindering.
\end{abstract}

Key words: Prevention, uterus cancer, swot analysis

\section{Introduction}

The cervical cancer is a cancer of the female genital whose impact has been drastically reduced through prevention implemented by the use of the Pap test in screening programs. In Italy, according to the Ministry of Health, they are diagnosed each year about 3,200 new cases of cervical cancer and 1,500 women die from this cancer [1]. The regional programs of screening for cervical cancer are within the essential levels of assistance, having been evaluated as proven interventions that can prevent in many cases the occurrence of such tumors and reduce, therefore, the incidence and mortality for these diseases.

Screening is a complex set of activities that includes, in addition to the test, information to the target population, the organization that facilitates access to the test and, for people who test positive, the preparation of diagnostic protocols, treatment and follow up.

Screening for cervical cancer is realized through the

Corresponding author: Giuseppe Andrea De Biase, epidemiologist statistical officer, health policy.
Pap test it should be done every three years in the age group 25-64 years, as stipulated by the regional program.

Adherence to screening in the Calabria region is lower than the national standard (respectively 33\% and 39\%, steps 2010-12) [2].

The data collected by the monitoring system "Passi" they say that the percentage of women who resort to early diagnosis of personal initiative is substantially similar to the other Italian regions. In Calabria, what makes the difference in coverage is generally the amount of people undergoing examination following a free test within an organized program [3].

Objective of this study is to seize, in the perception of the operators involved variously in screening system, the reasons for this phenomenon.

\section{Materials and Methods}

It was decided to use an analytical approach of SWOT, method investee with stakeholders that detects strengths (strenghts) and weaknesses (weaknesses) in the context of its analysis and the opportunities 
(opportunities) and threats ( threats) arising from the external environment faced by specific sectoral or territorial reality analyzed.

In any healthcare company we were invited all operators of screening centers, community services and hospital as well as the top executives of the Department of Health and Environment, of those who joined were acquired and analyzed in conjunction with the views expressed.

The information gathered through the SWOT have been plotted through the instrument of mental map, that, by providing the cataloging and organizing notes and ideas around a main concept, enabled us to bring together the strengths, weaknesses, opportunities and dangers of the regional screening.

Subsequently, after an encoding of the text, they were categorized into information units all common concepts and summarized in a single SWOT [4].

The strengths and weaknesses are internal to the context of analysis and are therefore modifiable through policy or specific interventions, opportunities and threats arising from the external environment and are not editable [5].

\section{Results and Discussion}

In May, 2013, they were carried out n. 9 meetings lasting an average of an hour and a half, at the epidemiological services, screening units and Health Protection Department who took part n.17 operators operating units screening, 1 family doctor, n. 15 midwives, n. 2 social workers, 2 pathologists, 4 gynecologists, 2 laboratory technicians, 1 responsible URP, 3 nurses, 1 manager, 1 in charge of counseling, 2 administration, 18 interviewers, n. 20 observers and n. 5 executives of the department of health protection, for a total of $n .84$ participants.

The SWOT analysis allowed to highlight the strengths and weaknesses of the regional system of screening of cervical cancer in the opinion of operators, as summarized in order of decreasing frequency in Figure 1.

Among the strengths it shows a large logistics organization capable of acting with presence in the region. Among the weaknesses identified most of the

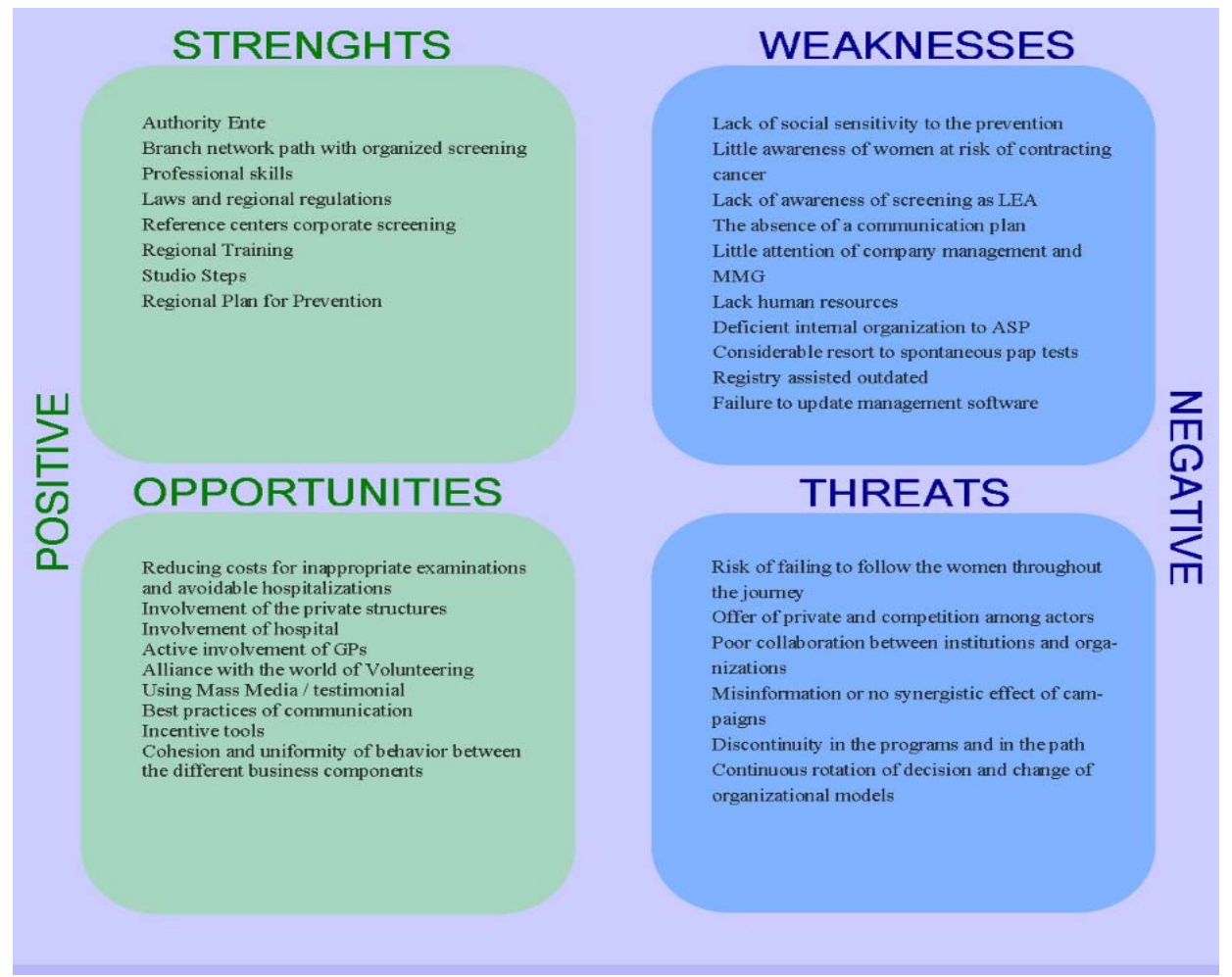

Fig. 1 Screening programs for cervical cancer-SWOT analysis with health care. 
stakeholders found in the population a lack of sensitivity to the issue of prevention, where despite the present knowledge that the same results in a reduction in cancer mortality could not pass the screen of cultural taboos especially atavistic inherent sense of decency.

Stakeholders point to a predictable lack of sensitivity in the same management company that, despite the fact that early detection of cervical cancer is a level of Essential Support, do not seem to assume consequential attitudes, allowing a widespread lack of human resources and Financial dedicated to screening and an inadequate facilities dispensing, accentuated by the limitations imposed by the current plan to reduce the deficit of health spending, which led to the blocking of expenditure on structural measures and equipment purchases in addition to the turn-over.

In particular, pathologists detect those deficiencies particularly in reading point of the tests that delegated to operational units of Pathology, due to the limited staff, not always performed within the three months required by current guidelines.

As for the critical issues of a systemic nature, it has emerged in the gaps identified nell'anagrafe assistititi, which do not allow a nationwide delivery of letters of invitation; the limited cooperation of General Practitioners in enlistment of eligible women; the widespread lack of motivation of health personnel involved in the screening, which determines poor action of advice, support and impetus; the discontinuity of information campaigns both locally and regionally.

Among the reported threats, the most important concern the current situation of competition with the private sector, not only and not so much for the competition in itself, but for the fact that the activities delivered by the private are almost totally divorced from the context of the programs screening.

\section{Conclusions}

The SWOT technique, precisely because of its structural peculiarities, is aimed at bringing about the activation of strategies to transform weaknesses into strengths and threats into opportunities. In this case, the study helped to identify some problems with respect to which we can assume the proposition of corrective actions [6].

They make it particularly suitable actions informational targeting women elected and decision-makers [7]. Compared to the first view to increase the culture of prevention, than the decision-makers, in order to increase awareness of the importance of screening, by providing documented evidence on the effects of reductive cancer mortality and, also, health costs.

Considering that it was found the prevalent use of two alternative sources registries SOGEI, fueled by Health Card system and the Register Regional, would be appropriate to adopt a single source of reference from which to extract the target population. The use of a single reference database would facilitate fact a cyclical process of "checking, cleaning and updating" of the data [8].

To overcome the widespread problem of the lack of human resources, the survey highlighted the opportunity to work on two different fronts: internally to companies, through the implementation of incentive systems for non-economic operators involved in screening (shifts, holidays etc.) with particular reference to the management of the operating units of pathology; externally, by integrating in the regional system studies gynecological individuals, hospitals, currently not involved, and the family doctors.

\section{References}

[1] Ministry of Health. 2013. "The cancer numbers in Italy, report 2013.” Accessed January 14, 2014.

[2] http://www.salute.gov.it/imgs/C_17_pubblicazioni_2250 _allegato.pdf. (in Italian)

[3] National Observatory screening (Ons). 2013. "Screening programs in Italy, short report 2013.” Accessed February 24, 2014.

[4] http://www.osservatorionazionalescreening.it/sites/defaul t/files/allegati/Screening_2014_web.pdf.

[5] (in Italian)

[6] Epicentro, The portal of epidemiology to public health. 
2013. “National report Passi 2010-2012: screening cervicale.” Accessed January 22, 2014. (in Italian)

[7] http://www.epicentro.iss.it/passi/rapporto2012/Screening Cervicale.asp. (in Italian)

[8] Rosati, I. 2013. Conceptual and mental maps maps. Tangram scientific editions. (in Italian)

[9] Hill, T., Westbrook, R. 1997. "SWOT Analysis: It's Time for a Product Recall”. Long Range Planning 30 (1): 46-52.

[10] Lawrence, G. 2009. The SWOT Analysis: Using Your
Strength to Overcome Weaknesses, Using Opportunities to Overcome Threats. Kick it, LLC.

[11] Epicentro, The portal of epidemiology to public health. 2013. "Communication and Empowerment in screening programs.” Accessed April 2, 2014.

[12] http://www.epicentro.iss.it/discussioni/screening/Federici -orig.pdf. (in Italian)

[13] Prabhakaran, B. 1997. Multimedia Database Management Systems. The Springer International Series in Engineering and Computer Science, 375. 